\title{
Stratégies des ménages kanak : hybridations entre logiques marchandes et non marchandes
}

\author{
Séverine Bouard ${ }^{1}$, Jean-Michel Sourisseau ${ }^{2}$ \\ a Doctorante en géographie, IAC-CIRAD, CIRAD, 73 avenue Jean-François Breton, 34398 Montpellier cedex 5, France \\ b Socioéconomiste, IAC-CIRAD, Centre de recherche du Nord, BP 6, 98825 Pouembout, Nouvelle-Calédonie
}

\begin{abstract}
On sait bien que la question du développement est ambiguë et qu'elle reflète d'abord les valeurs d'un certain système de pensée, celui du monde occidental. Ainsi, la société kanak de Nouvelle-Calédonie ne rentre pas dans les catégories que nous nous sommes données pour concevoir les politiques publiques de développement territorial et leurs instrumentations : que veulent dire «ménage », « ruralité », « agriculture » dans ces sociétés? Quels sens peut-on donner à des notions comme «marchand » et «non-marchand » quand ces dimensions s'hybrident en permanence dans des produits qui servent à la fois à se nourrir et à faire face à ses obligations coutumières et dont on peut vendre le surplus? Une vieille question pour les anthropologues qui est au cœur de l'actualité du développement durable.
\end{abstract}

La Rédaction

\section{Mots-clés :}

logiques marchandes et non marchandes ; développement territorial ; multifonctionnalité ; pluriactivité ; Nouvelle-Calédonie

\section{Keywords:}

trade and non-trade concerns; pluriactivity; multifunctionality; local policies; New-Caledonia
Résumé - Inscrit dans le cadre de l'anthropologie économique et de la nouvelle sociologie économique, cet article propose une grille de lecture intégrant les liens entre les sphères économique et sociale pour étudier les stratégies des ménages ruraux. Cette vision offre des outils d'analyse des stratégies des acteurs reposant sur la reconnaissance de l'enchâssement des relations économiques dans les relations sociales. L'analyse d'enquêtes réalisées auprès de ménages ruraux kanak en Nouvelle-Calédonie souligne que le recours généralisé à la pluriactivité, la diversité des pratiques agricoles et les choix de valorisation des produits ruraux résultent d'une hybridation entre logiques marchandes et non marchandes. Cette approche des stratégies des acteurs éclaire de façon originale les phénomènes de recomposition territoriale à l'œuvre en Nouvelle-Calédonie aujourd'hui.

\begin{abstract}
Linkages between the economic and the social spheres: strategies of the Kanak rural households. Our study results from an action-research project accompanying a development project in New Caledonia. This paper proposes an analytical framework based on the linkages between the economic and social spheres to investigate the strategies of rural households. We plan to reconsider the notions of trade and non-trade concerns as detailed in recent studies in economic anthropology and new economic sociology. This approach enabled us to formalize tools based on the recognition of the "embeddedness" of economic relations in social relationships to analyze actor strategies. The Kanak rural population offers a perfect illustration of the interest of viewing the economic and social organization in this way. Kanak households are undergoing a recent and significant economic development, which means extended integration into the market, but which also encounters some specific social rules in an ambiguous way. Based on the results of inquiries in Kanak households on the East coast of New Caledonia, the article shows that pluractivity, the diversity of agricultural practices as well as of uses of rural products result from hybridization between trade and non trade logics. Finally, the article shows that such an approach to actor strategies highlights in an original way the phenomena of territorial reorganization in New Caledonia today.
\end{abstract}


Cet article ${ }^{1}$ propose d'analyser les stratégies de ménages à partir d'une grille intégrant les liens entre les sphères économique et sociale, puis d'en tirer des enseignements pour alimenter la réflexion sur les approches de développement local.

Dans une première partie, nous revisiterons les notions de marchand et de non-marchand, en mettant en évidence le caractère réducteur d'une opposition stricte de ces deux concepts. Au contraire, nous tenterons de montrer que le marchand et le non-marchand sont présents dans chaque acte économique, qu'ils s'articulent pour réguler, ensemble, les échanges de biens et de services et, par suite, la vie économique des ménages ruraux. Cette vision nous permettra de formaliser des outils d'analyse des stratégies des acteurs, reposant sur la reconnaissance de l'enchâssement des relations économiques dans les relations sociales.

Le monde rural calédonien, en particulier kanak, offre une parfaite illustration de l'utilité d'une telle lecture de l'organisation économique et sociale. Les ménages y connaissent en effet un développement économique récent et conséquent, qui suppose une plus grande insertion dans le marché, mais qui rencontre des règles sociales privilégiant souvent, en première analyse, les coordinations non marchandes. L'analyse des enquêtes réalisées auprès de ménages ruraux sur la côte est de la province Nord montre que le recours généralisé à la pluriactivité, la diversité des pratiques agricoles et les choix de valorisation des produits ruraux résultent bien d'une hybridation entre logiques marchandes et non marchandes.

\section{Marchand, non-marchand : définitions et implications analytiques}

Le marchand et le non-marchand apparaissent intuitivement comme deux notions diamétralement opposées. On peine à trouver une expression pour mieux qualifier le second autrement qu'en négation du premier. Les apports de l'anthropologie économique et, plus récemment, de la nouvelle sociologie économique montrent au contraire que les deux notions sont en réalité difficilement dissociables.

Polanyi et al. (1957) définissent les relations économiques comme un processus social : la production et la distribution de biens et de services peuvent prendre des formes diverses selon l'univers dans lequel elles opèrent. Ces auteurs formalisent ainsi le concept d'enchâssement (embeddedness) de l'économie dans des formes de coordination avant tout sociale et culturelle. Ils décrivent trois motivations qui animent l'économie : la maximisation du profit, à l'origine de l'échange marchand ; le partage entre

${ }^{1}$ Ce travail a fait l'objet d'une communication au colloque CORAIL "Territoires et patrimoine en Océanie ", Nouméa, décembre 2005. les membres d'une même communauté entretenant les formes hiérarchiques préexistantes, à l'origine de la redistribution des biens et services ; la solidarité agissant entre des groupes d'individus mus par des obligations morales, à l'origine d'échanges fondés sur la réciprocité. En combinant ces trois moteurs de l'implication des individus dans l'échange de biens ${ }^{2}$, des formes différenciées de régulation des activités économiques coexistent (Castel, 2003).

Granovetter (1985) mobilise quant à lui la notion d'enchâssement pour montrer l'importance des jeux sociaux dans l'accès aux ressources et aux moyens de production. Il insiste sur la nécessité de déconstruire les représentations du marché de concurrence pure et parfaite : la mise en marché n'est qu'une utilisation parmi d'autres des produits de l'activité économique et cette forme de l'échange ne peut être comprise par les seuls mécanismes de la concurrence. Il convient de considérer les interactions entre échange marchand et relations interpersonnelles non marchandes, qui ont leur logique propre et sont explicatives des motivations et des objectifs des échanges (Le Velly, 2002).

D'autres travaux mettent l'accent sur les hybridations entre le marchand et le non-marchand, en considérant que ces deux notions n'ont de valeur que théorique (Groupe Polanyi, 2008). Ce sont des idéaux types, la réalité exprimant une infinité de combinaisons entre ces extrêmes conceptuels. Chaque situation observée témoigne alors d'un certain niveau d'intégration au marché et, conjointement, d'un certain degré d'identification sociale ; il est possible de lire les relations d'échange comme la résultante d'un processus d'abstraction marchande, qui tire ces relations vers le marché, et d'un processus d'identification sociale, qui les en éloigne.

L'échange marchand se caractérise par l'anonymat des contractants : acheteur et vendeur n'ont d'autres caractéristiques que celles de leur fonction d'acheteur et de vendeur. Ils opèrent hors de tout groupe social, comme des acteurs strictement individuels et autonomes. À l'opposé, le non-marchand correspond à une vision strictement holiste. Chaque individu est membre d'un groupe dont la destinée prime sur les aspirations individuelles. Les relations entre les individus reposent sur l'interconnaissance, le respect de règles communes historiquement construites et se déclinent en obligations et en droits. L'identification sociale des individus ne repose pas sur des principes égalitaires, mais sur des hiérarchies sociales et des inégalités. En retour, la communauté garantit à chacun de ses membres, à des degrés divers, reconnaissance

\footnotetext{
${ }^{2}$ On pourrait aussi, sous certaines conditions, ajouter au modèle la dimension rituelle, et notamment la mise en relation entre la sphère des morts et la sphère des vivants. Dans la plupart des sociétés mélanésiennes, de nombreux échanges ont pour support cette mise en relation et peuvent, éventuellement, compléter et renforcer le partage et la solidarité.
} 
sociale, accès à des formes de solidarité et notamment aux circuits d'échanges réciproques. La disponibilité et la mobilisation des moyens de production, ainsi que l'utilisation des biens et services produits, sont contrôlées collectivement.

En réalité, des processus antagonistes mais complémentaires influencent le positionnement des échanges entre ces deux pôles idéels. Un processus d'abstraction marchande tend à renforcer l'intégration de l'individu au marché et sa sensibilité aux signaux de prix, à l'extraire de ses réseaux de relations interpersonnelles. Cela se traduit par une monétarisation croissante des échanges et la recherche d'accumulation productive pour en augmenter l'intensité capitalistique. Mais, en même temps, toute communauté génère ses propres mécanismes de maintien de son identité en opposition/résistance aux logiques marchandes, notamment en modulant la carte des droits et des obligations et les mécanismes de sanction qui y sont attachés (ibid.). Ces processus d'abstraction et d'identification agissent conjointement, s'hybridant mutuellement, sans que l'un domine l'autre a priori. Il faut alors s'intéresser aux conditions de l'enchâssement de l'économique dans le social, chercher à comprendre en quoi les forces sociales favorisent ou contraignent l'échange marchand (Le Velly, 2002). C'est ce cadre théorique que nous proposons de mobiliser pour analyser les stratégies des ménages ruraux kanak.

\section{Stratégies des ménages kanak de la côte est de la Nouvelle-Calédonie}

\section{Contexte général de la Nouvelle-Calédonie et de la zone d'étude}

La Nouvelle-Calédonie, archipel de $19000 \mathrm{~km}^{2}$ et peuplé de 240000 habitants, est située à $1500 \mathrm{~km}$ à l'est des côtes australiennes, dans l'ensemble mélanésien. Elle bénéficie d'un statut spécifique, produit des accords politiques de Matignon-Oudinot (1988) et de Nouméa (1998), qui entend répondre aux revendications indépendantistes qui se sont violemment exprimées dans les années 1980. Outre une marche programmée vers l'autonomie, ce statut instaure trois provinces dotées d'importantes compétences. En province Nord (Fig. 1), dans laquelle se situe notre terrain d'étude, la majorité de la (faible) population est kanak (32246 habitants, soit 77,9\%, en 1996, derniers chiffres disponibles). Après des décennies de marginalisation liée à la colonisation de peuplement européen, ces changements politiques récents ont considérablement amélioré l'insertion des Kanak dans l'économie. Ils ont ainsi accéléré les phénomènes d'hybridation entre les structures sociales fondatrices du monde mélanésien et celles issues de la colonisation.

La société kanak est organisée sur la base d'une unité sociale et spatiale particulière : le clan. Celui-ci se définit comme un groupe humain composé de plusieurs familles ou lignages (qui ont chacun leur nom patronymique, transmis de manière patrilinéaire), descendant d'un ancêtre commun. Ses membres vivent sur une terre qu'ils gèrent en communauté selon des rapports sociaux hiérarchiques bien définis. Partant de cette identification par la terre, la manifestation des relations sociales entre individus et groupes sociaux dépend du degré de parenté, de l'âge de l'individu et du caractère d' " aîné », ou " premier né », selon un principe de liens de parenté ascendante de chaque individu au sein de chaque lignage et de chaque lignage au sein du clan (Godin, 2000). La coutume est l'ensemble des règles non écrites qui régissent l'équilibre social des Kanak; elle organise en particulier les dons et contre-dons, gestes qui marquent tout acte social au premier rang desquels les mariages et les deuils - et tracent les contours des alliances entre les clans. Pour Haudricourt (1964), la société kanak est le produit d'une civilisation de l'igname, produit le plus symbolique des échanges coutumiers, si bien que son cycle de culture rythme le calendrier social.

Ces éléments sont particulièrement prégnants dans les communes de Touho, Poindimié et Ponérihouen, concernées par l'analyse. Situées sur la côte est de la province Nord, loin des centres miniers forgeant la richesse de la Nouvelle-Calédonie et du bassin d'emploi du chef-lieu de province (le pôle Voh-Koné-Pouembout de la côte ouest), elles sont très majoritairement peuplées par des Kanak pour lesquels, nous le verrons, les circuits d'échanges restent très vivants. Les trois villages ${ }^{3}$ concentrent commerces et services privés et publics, tandis que les implantations humaines se dispersent le long de la côte et dans les vallées, dans de très nombreuses tribus présentant des configurations d'accessibilité très diverses (Fig. 1). Ces tribus, créées par l'administration coloniale à la fin du XIXe siècle après le cantonnement des Kanak dans les réserves indigènes, constituent aujourd'hui une réalité sociologique significative. Les cadres hiérarchiques de la tribu, anciennement relais de l'administration coloniale, se superposent à l'autorité des clans, parfois dans des relations contradictoires (elles englobent souvent, mais aussi parfois séparent les clans), mais la tribu est aujourd'hui un espace de résidence et d'appartenance sociale qui fait sens.

\section{Les bases empiriques de l'analyse}

Les résultats qui suivent sont issus d'enquêtes réalisées auprès de ménages kanak, dans le cadre de la mise

\footnotetext{
Les villages sont les « centres urbains » des communes, ils sont principalement habités par la population d'origine européenne. Les tribus, qui sont des hameaux habités par les Kanak, sont dispersées autour du village sur l'ensemble du territoire communal.
} 


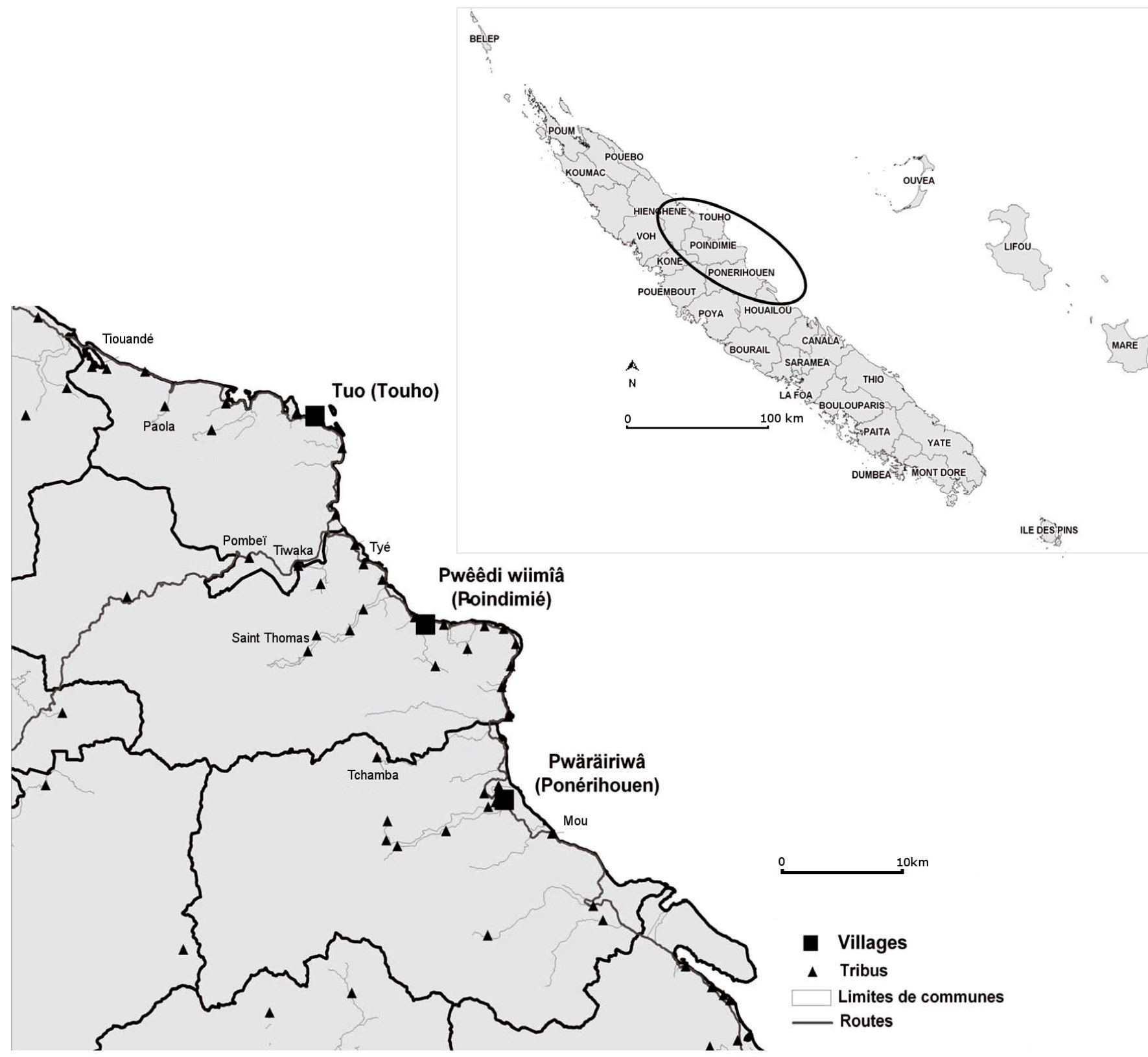

Fig. 1. Zone d'étude et tribus enquêtées.

en place par l'Institut agronomique néo-calédonien d'un dispositif de suivi participatif de l'opération groupée d'aménagement foncier (OGAF) touchant les communes de Touho, Poindimié et Ponérihouen (Bouard et al., 2006). Ce suivi participatif s'inspire de la démarche de gestion progressive autonome développée au Sénégal (d'Aquino, 2009), partiellement adaptée au contexte calédonien. L'OGAF est une opération de développement local qui vise au départ à financer des projets agricoles. L'objet du suivi participatif était de juger des innovations développées, mais aussi de l'adéquation des mesures mises en place avec les dynamiques concrètes des ménages concernés. Outre l'expérimentation d'outils novateurs d'animation participative (Bouard et d'Aquino, 2007), une approche spécifique des stratégies microéconomiques et microsociales des ménages ruraux en tribu a été conduite.

Partant des acquis de la recherche sur le fonctionnement socioéconomique du monde rural kanak (Barrau, 1956 et 1983 ; Leblic, 1993 et 2002 ; Djama, 1999), les entretiens réalisés portaient sur les structures familiales, les activités exercées et leurs combinaisons. L'unité d'observation choisie, le ménage, correspond ici à un foyer comprenant le chef de ménage et ses dépendants vivant avec lui, c'est-à-dire, schématiquement, prenant leurs repas au sein du foyer. Cette unité, inscrite dans d'autres unités plus larges, susceptibles de contrôler partiellement les moyens de production (le clan en particulier), apparaît 
Tableau 1. Présentation de l'échantillon enquêté.

\begin{tabular}{|c|c|c|c|c|c|}
\hline Communes & $\begin{array}{l}\text { Tribus } \\
\text { enquêtées }\end{array}$ & $\begin{array}{c}\text { Nbre de ménages } \\
\text { en } 1996\end{array}$ & $\begin{array}{c}\text { Nbre d'habitants } \\
\text { en } 1996\end{array}$ & $\begin{array}{c}\text { Nbre de ménages } \\
\text { enquêtés }\end{array}$ & $\begin{array}{c}\text { \% de ménages. } \\
\text { enquêtés }\end{array}$ \\
\hline \multirow[t]{3}{*}{ Touho } & Paola & 51 & 251 & 6 & $11,8 \%$ \\
\hline & Pombeï & 15 & 75 & 3 & $20,0 \%$ \\
\hline & Tiouandé & 29 & 124 & 2 & $6,9 \%$ \\
\hline \multirow[t]{3}{*}{ Poindimié } & Saint-Thomas & 63 & 288 & 7 & $11,1 \%$ \\
\hline & Tiwaka & 23 & 135 & 10 & $43,5 \%$ \\
\hline & Tyé & 72 & 374 & 12 & $16,7 \%$ \\
\hline \multirow[t]{3}{*}{ Ponérihouen } & Embouchure & 55 & 202 & 3 & $5,5 \%$ \\
\hline & Mou & 39 & 187 & 6 & $15,4 \%$ \\
\hline & Tchamba & 73 & 292 & 12 & $16,4 \%$ \\
\hline Total & & 420 & 1928 & 61 & $14,5 \%$ \\
\hline \multicolumn{2}{|c|}{ Toutes les tribus des 3 communes } & 1568 & 7291 & 61 & $3,9 \%$ \\
\hline
\end{tabular}

pertinente pour juger des stratégies d'activités et de leur mise en œuvre. Les décisions techniques et d'utilisation des produits sont, certes, le plus souvent partiellement encadrées et codifiées au sein du clan, mais elles relèvent bien des individus et sont dimensionnées au sein de l'unité de résidence et de consommation.

La ruralité est ici entendue comme un espace de faible densité de peuplement, en opposition aux espaces urbains. Dans le cas de la Nouvelle-Calédonie, pays polarisé, tout ce qui n'est pas Nouméa est communément appelé «brousse » et peut être considéré comme relevant du rural. À cette dimension démographique s'ajoute une dimension identitaire, qui s'exprime par le lien symbolique à la terre cultivée et les liens entretenus dans un espace de proximité et d'interconnaissance forte, et qui est pensée elle aussi en opposition à l'urbain. Enfin, nous avons choisi de conserver le terme d'agriculture, alors que lui est souvent préféré celui d'horticulture (Haudricourt, 1964 ; Leblic, 2002). Nous l'entendons dans une acceptation large de production végétale, sans présager de l'utilisation marchande, alimentaire ou symbolique des productions.

Les enquêtes détaillent la composition du ménage, puis, pour chaque individu, l'âge, le niveau d'études, les activités exercées et les modalités de leur combinaison, les revenus dégagés, les formes et niveaux de la consommation, etc. Pour les produits agricoles, les produits pêchés et chassés, nous avons tenté de reconstruire avec la ou les personnes enquêtées quelles étaient les parts consommée, donnée et vendue. Une attention particulière a été portée aux fonctions non marchandes assignées aux activités rurales : dons et autoconsommation de produits, marquage foncier, affirmation de sa place dans la communauté. Les enquêtes se sont également intéressées aux stratégies globales des ménages et des individus qui les composent, et aux liens existant entre elles et entre ces individus.

Les enquêtes ont été réalisées auprès de 61 ménages répartis dans les 3 communes de la zone de l'OGAF, côte est (Tab. 1). Ces ménages ont été choisis parmi 9 tribus, retenues sur les conseils des services techniques provinciaux pour représenter la diversité des situations géographiques (chaîne montagneuse et proximité d'un axe routier) [Fig. 1]. Tous les ménages enquêtés résident en tribu. Ils sont composés en moyenne de 5,5 personnes, $36 \%$ ont entre 4 et 6 membres. Pour comparaison, les ménages de la province Nord comptaient en moyenne 4 personnes seulement en 2004 (ISEE, 2005). Si les fonctionnements concrets des ménages mis en lumière ont un domaine de validité limité de par la taille de l'échantillon et de la zone d'enquête, les principes méthodologiques testés et les grands mécanismes explicatifs des dynamiques rurales révélés ont une portée plus générale. Ils recoupent partiellement les travaux de Freyss (1995) et de Guiart (1999), qui, avec des visions respectivement macroéconomique et ethnographique, insistent sur le poids du non-marchand dans les dynamiques économiques et sociales.

\section{Des systèmes d'activités complexes et souples}

Le revenu monétaire moyen des ménages enquêtés s'élève à $660,70 € /$ mois, ce qui est bien inférieur au salaire minimum garanti (876 €/mois en 2005) et au salaire agricole minimum garanti (725 €/mois). Les enquêtes montrent que ces revenus monétaires sont issus de plusieurs activités : agriculture, chasse, pêche et salariat (Fig. 2). La pluriactivité est très présente et semble diriger les stratégies d'acquisition de revenus. Ce phénomène est inscrit dans l'histoire du pays; les opportunités d'emplois directs, indirects et induits de la mine, de l'administration et/ou des opérations de développement agricole traversent les trajectoires des acteurs locaux, en s'articulant avec le maintien d'une agriculture conservant ses dimensions alimentaire et coutumière. Depuis les débuts de l'intégration marchande, la gestion de plusieurs activités est significative des choix de vie des ménages. 


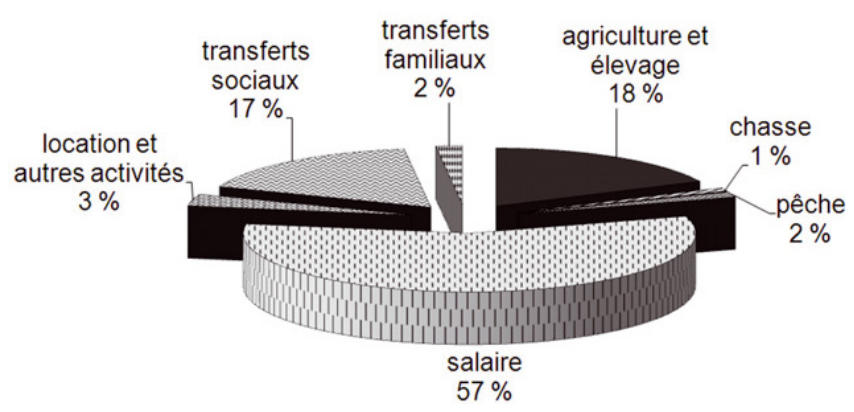

Fig. 2. Contribution des différentes activités au revenu monétaire moyen de l'échantillon.

La figure 2 confirme la vitalité des principales activités tribales, malgré leur faible poids marchand ( $20 \%$ des revenus monétaires). L'agriculture est la principale occupation des ménages de notre échantillon, qui y consacrent en moyenne 134 jours homme/an. Hormis pour quelques rares $\operatorname{cas}^{4}$, la pratique de l'agriculture vise certes à obtenir des revenus d'appoint, mais plus encore à fournir des produits alimentaires de base et des produits symboliques à donner. Elle obéit ainsi davantage à des logiques sociales et culturelles qu'à des logiques productives pour accroître un revenu monétaire (Fig. 3). La pêche, en mer comme en rivière, est pratiquée par près de $80 \%$ des ménages, à hauteur de 68 jours homme/an. La chasse intéresse quant à elle près des deux tiers des hommes enquêtés, mais elle est moins mobilisatrice en temps (27 jours homme/an). Comme pour l'agriculture, les logiques non marchandes dominent dans la valorisation des produits de la pêche et de la chasse.

En accord avec les résultats disponibles sur les systèmes d'activités dans le monde kanak (Mercoiret et al., 1999; Sourisseau, 2008), le salariat est un élément structurant des stratégies d'acquisition de revenus : $80 \%$ des ménages enquêtés ont eu une activité salariée dans l'année, quelles qu'en soient la durée et la nature. Ces emplois sont majoritairement non qualifiés : construction (cases pour des cérémonies, maisons individuelles...), entretien des routes, etc. ; ils concernent pour $69 \%$ des contrats à la tâche et des CDD de moins d'un an.

L'absence de spécialisation des systèmes d'activités peut s'analyser comme une option par défaut, du fait d'un accès limité à un emploi stable ou de contraintes trop fortes rencontrées pour créer sa propre entreprise. Mais elle peut également s'interpréter comme un choix privilégiant la prévention contre les risques d'un environnement économique et social instable. De par sa valeur symbolique, en particulier pour ce qui concerne la culture des ignames et des taros pour les échanges coutumiers, l'agriculture participe de cette prévention ; l'abandonner

4 On compte un à deux agriculteurs kanak par commune correspondant au profil type de l'agriculteur «professionnel».

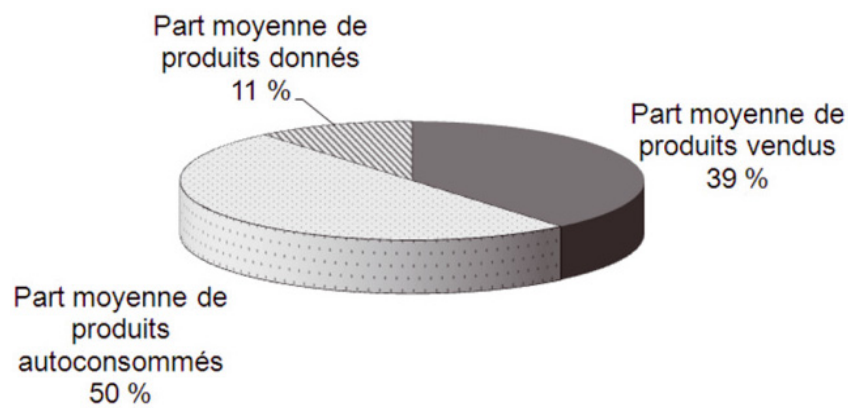

Fig. 3. Valorisation moyenne des produits de l'agriculture, de la chasse et de la pêche.

au profit d'un emploi qui augmenterait le revenu direct n'est pas forcément souhaitable.

Dans une perspective strictement économique et individuelle, les pratiques observées d'accès à l'emploi salarié sont difficilement compréhensibles. Des CDI sont rompus sans raison objective apparente : «Une nièce m'avait parlé de ce poste de maçonnerie au village... Je suis resté 6 mois mais après je ne voulais plus. Je ne voulais pas du travail et ça ne me plaisait pas... Je n'aime pas trop la maçonnerie, je préfère la mécanique, j'ai arrêté » (A. Seuchi, 13 avril 2005). Dans le même temps, certaines personnes n'hésitent pas à quitter leur tribu quelques mois pour saisir une opportunité d'emploi, notamment vers Nouméa. Ces logiques alternatives prennent tout leur sens lorsque sont considérées l'insertion partielle des individus au marché et la faiblesse de leurs besoins monétaires courants. En revanche, les individus ont des dépenses ponctuelles à assurer et n'ont recours au salariat que dans ces occasions. Le contrat peut donc être arrêté une fois que la dépense concernée est réalisée. Par ailleurs, la gestion de l'emploi revêt parfois une dimension collective : des allers et retours entre la sphère domestique et le salariat sont souvent organisés dans le réseau familial élargi. Une relation personnelle, familiale ou de voisinage peut proposer un emploi. L'accepter permet alors d'entretenir un réseau et de renforcer les liens sociaux.

En écho aux systèmes d'activités, la complexité des systèmes de production est remarquable. Pour notre échantillon, on compte en moyenne 2,7 parcelles comportant 3,1 espèces différentes et plus de 8 espèces par ménage $^{5}$. Les tubercules dominent (ignames, taros d'eau), mais en moyenne moins de 600 pieds sont plantés par ménage, loin des seuils de viabilité d'une exploitation marchande. Les dépenses moyennes des ménages en intrants sont très faibles $(119 € / \mathrm{an})$. Les produits les plus couramment utilisés sont les désherbants, parfois de l'insecticide

\footnotetext{
${ }^{5}$ Ces chiffres sont probablement sous-estimés : certains fruitiers isolés n'ont pas toujours pu être recensés, de même que certaines plantes caractéristiques du jardin horticole mélanésien (canne à sucre, maïs, pois d'angole, etc.), présentes en faibles quantités.
} 
et du carburant pour le débroussaillage. Beaucoup de ménages ont très peu de matériel, tant pour cultiver que pour pêcher ou chasser; la valeur moyenne des équipements est inférieure à $417 €$.

Les activités rurales suivent des techniques anciennes et éprouvées, très peu coûteuses si le travail familial n'est pas comptabilisé. On note cependant, partant de ces savoir-faire de base, une différenciation croissante des pratiques. Les systèmes techniques s'adaptent selon les opportunités, peu prévisibles, d'accès à un travail salarié. Ainsi, les hauteurs de billons, le tuteurage ou la gestion des adventices pour les champs d'igname offrent des combinaisons diverses, correspondant à des temps de travail pouvant varier du simple au double sur une même opération technique (Gaillard et Sourisseau, 2009).

En fin de compte, l'accès à un salaire ne se fait pas strictement au détriment des activités rurales «traditionnelles » que sont la pêche, la chasse et l'agriculture. «J'ai un contrat pour construire une maison [...] je rattrape le travail à la ferme [second petit contrat] après, puis je vais sur mon champ » (J. Poeda, 14 juillet 2005). La souplesse des systèmes permet une répartition des tâches au sein du ménage. Le caractère extensif et la plasticité des activités «tribales » permettent aussi, et c'est plus souvent le cas, leur combinaison avec un emploi salarié.

\section{Des stratégies de valorisation des produits entre marché et solidarité}

La valorisation des produits ruraux (issus de l'agriculture, de la pêche et de la chasse) illustre parfaitement la logique de l'enchâssement (Fig. 3). Concernant près de $10 \%$ des productions agricoles des ménages enquêtés, mais plus du quart des produits de la chasse et de la pêche, le don répond clairement à des logiques sociales plutôt que marchandes et sous-tend le raisonnement stratégique de production et de valorisation des produits. Le don de biens agricoles, alimentaires et symboliques (igname) est une obligation coutumière. Les volumes dépendent des sollicitations cérémonielles (naissance, mariage, etc.), variables d'une année sur l'autre. Ces cérémonies, et les échanges qui les rythment, sont des actes sociaux déterminants qui participent au processus d'identification sociale. Il est difficile de s'y soustraire et la substitution par un don monétaire n'est pas toujours admise, d'autant qu'aucun prix n'est associé à ces biens. Les dons sont faits à des parents ou alliés, qui retourneront à terme ce geste ; ils correspondent à un échange de réciprocité, voire de redistribution selon les acteurs concernés.

La consommation du ménage mobilise près de la moitié des volumes de production de l'agriculture et de la pêche, plus encore pour la chasse. Elle reste donc l'utilisation principale des productions rurales. Dans un contexte de prix élevés des aliments de base, cela a une incidence très forte, et trop souvent négligée, sur les budgets des ménages, mais il serait limitant de n'y voir qu'un acte économique. La pratique agricole pour la consommation est aussi symbolique $d^{\prime}$ 'un mode de vie, d'une affirmation de la mise en valeur des terres de son clan, et donc d'une inscription sociale dans sa communauté.

Les ventes représentent $39 \%$ des valorisations des produits agricoles. Cela témoigne d'une réelle intégration aux circuits marchands, même si les quantités concernées sont très éloignées des standards de la spécialisation. Une analyse fine des circuits de commercialisation des produits agricoles montre néanmoins que ces actes de vente ne sont que partiellement régis par des coordinations marchandes.

En réponse à la faiblesse constatée des circuits de collecte primaire, les ménages kanak ont ainsi initié des modes hybrides de commercialisation : les marchés de proximité. On distingue ici les marchés communaux, aménagés dans le centre du village de la commune, et les marchés en tribu. Les volumes échangés sont faibles : de quelques dizaines ou centaines de kilos par semaine pour les tubercules, les bananes et les fruits, selon la saison et la taille du marché. La participation est basée essentiellement sur deux motivations : le désir de rencontrer d'autres personnes et celui de faire connaître et redistribuer ses produits locaux. Les prix pratiqués peuvent être différents selon la capacité financière de l'acheteur (touriste, connaissance, client fidèle, etc.). C'est donc la nature de la relation sociale qui détermine la valeur de la transaction et non pas uniquement la valeur marchande du produit. Ces marchés sont de nouveaux espaces de valorisation économique des produits de terroir, de reconnaissance et valorisation des savoirs locaux, mais surtout de renforcement de la cohésion sociale (Sabourin et Tyuienon, 2007).

Les étals de bord de route présentent aussi un caractère hybride entre marchand et non-marchand. Le vendeur laisse ses produits en exposition libre, avec un prix fixe affiché; l'acheteur dépose la somme exacte et emporte ses achats, sans contact avec le producteur. Ce fonctionnement tend en première analyse vers une plus grande autonomisation des acteurs, plus d'anonymat, et donc plus de concurrence. Dans les faits, les passants s'arrêtent en général aux étals de personnes «connues » et reproduisent les coordinations rencontrées sur les marchés de proximité, fondées sur la solidarité et le renforcement des liens sociaux (ibid.). De plus, le mode de paiement repose sur la confiance. Ce système offre enfin le maximum de souplesse, et donc d'adéquation avec les logiques adaptatives des systèmes d'activités : le producteur n'alimente son étal que lorsqu'il en a la possibilité et ne consacre pas de temps à la vente.

Les ventes à des particuliers qui passent des commandes directement aux producteurs se sont aussi développées. Les commandes évitent à l'agriculteur d'importants efforts et coûts de démarchage, négociation et 
transport; elles concernent aussi la pêche et la chasse : «Je vais chasser le samedi, mais pas tous les samedis [...] ça dépend s'il y a des commandes [...] une fois par mois, oui » (A. Goroatu, 7 juin 2005). Les foires offrent aussi l'occasion de faire quelques «coups ». Supposant souvent une organisation collective et une certaine anticipation, la foire permet de partager (ou de se faire subventionner) certains coûts et offre un lieu supplémentaire de vente.

Les structures collectives prenant directement en charge la commercialisation des produits (coopératives, certaines associations de producteurs) pâtissent de la vivacité de ces circuits. Malgré la mise en place de nouvelles formes de contrats pour tenir compte des difficultés des acteurs locaux (écoulement et coûts de transport), leurs prix sont peu compétitifs et les conditions offertes moins souples que celles des circuits de proximité. Cela explique la faible part occupée par la commercialisation collective (Fig. 4).

Chaque ménage utilise en moyenne près de quatre modes de commercialisation différents, pour des recettes moyennes de $1676 €$ /an/ménage. La faiblesse de ces recettes contraste bien avec la diversité des lieux de vente et le temps consacré aux activités rurales. Seule la prise en compte de fonctions non marchandes de l'activité productive explique cet apparent paradoxe.

\section{Liberté individuelle versus contrôle social des moyens de production}

Plus largement, les enquêtes montrent que, si les individus ont tout pouvoir de décision dans l'exercice des activités qu'ils développent (choix techniques et allocations des facteurs de production), ils doivent négocier avec les membres de leur clan les conditions d'utilisation des moyens de production, notamment la terre, ainsi que la valorisation des fruits de leur travail.

Le contrôle collectif de l'activité économique reste une réalité, même s'il ne se traduit pas forcément par des travaux communs. Ce principe offre une autre piste d'interprétation des logiques non marchandes qui se déploient dans certaines activités, dont l'agriculture. Ainsi, l'accès à la terre n'est généralement pas problématique pour mettre en valeur des surfaces limitées, tournées vers la consommation et les petites ventes de proximité, mais il peut le devenir lorsque l'activité agricole est davantage marchande et concerne une plus grande surface. Mais ce contrôle n'a pas que des effets négatifs ; si les légitimités sont respectées et que l'individu concerné parvient à justifier de la cohérence de son projet avec les dynamiques de son clan et de sa tribu, le contrôle collectif peut devenir un appui. Un projet de type marchand sera alors consolidé par des mécanismes relevant a priori de l'identification sociale. Enfin, des modalités de gestion de cette hybridation se mettent en place, qui permettent une coexistence négociée : «On fait la part des choses [...], il faut

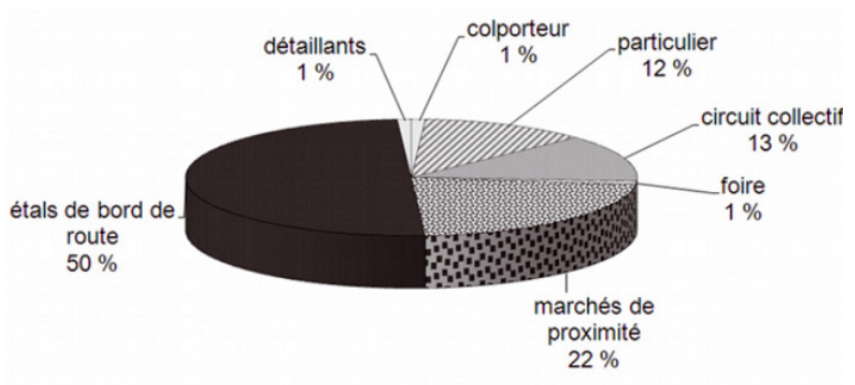

recettes (somme de toutes les transactions par lieu de commercialisation)

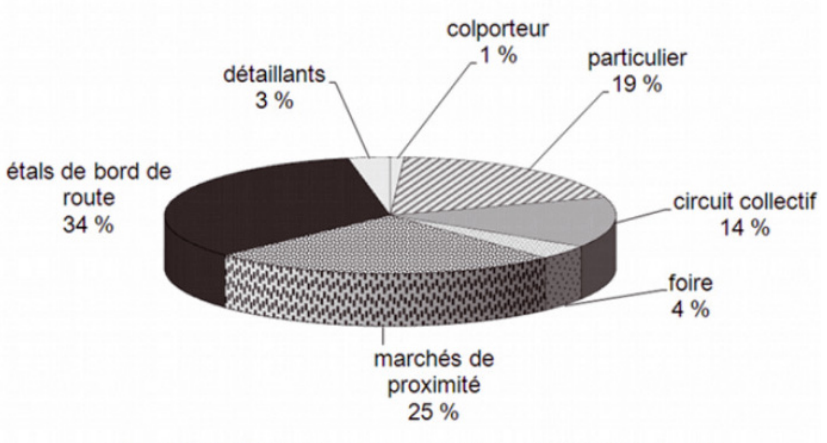

nombre de fois oủ le lieu de commercialisation est choisi dans l'année

Fig. 4. Poids des différents modes de commercialisation des produits ruraux.

gérer entre les obligations sociales et le travail » (L. Waka, 18 août 2005).

Les modes de valorisation des produits agricoles sont aussi encadrés par les règles communautaires. La préférence pour la commercialisation de proximité traduit le poids des relations sociales dans les échanges marchands. Nous avons vu que ces circuits reposent sur l'interconnaissance des différents acteurs et que la construction des prix n'obéit pas aux lois de l'offre et de la demande. Comme pour l'accès aux moyens de production, le choix de pratiques plus anonymes, régulières et rémunératrices nécessite un aval collectif.

L'individu ne fait donc pas ce qu'il veut, et ces exemples issus de l'activité agricole pourraient se décliner pour les autres secteurs économiques. Dans une approche économique et individuelle, cette situation est souvent perçue comme une contrainte puisqu'elle limite les possibilités d'insertion marchande des individus, mais l'analyse montre plutôt que l'équilibre entre marchand et non-marchand se conçoit dans la recherche globale d'une cohérence entre l'organisation sociale communautaire et les contraintes et opportunités marchandes.

\section{Pour une lecture renouvelée des stratégies des ménages ruraux}

À l'issue de cette analyse empirique, il est possible de mieux caractériser les processus d'abstraction 
marchande et d'identification sociale à l'œuvre sur notre terrain d'étude.

\section{Quelques traits marquants des processus d'abstraction marchande}

Les facteurs facilitant l'intégration marchande et les logiques individuelles tournées vers la recherche de profit sont principalement liés au développement du salariat, combiné à des changements des modes de consommation. C'est surtout sous l'action de ces phénomènes que la monétarisation de l'économie des ménages s'opère. Le salariat suppose une plus grande mobilité des personnes, qui peut à terme créer des ruptures de certains liens sociaux. La survalorisation de biens de consommation relevant du monde occidental induit également une individualisation des relations économiques, ou tout au moins un repli sur des entités collectives plus restreintes.

La diversification des modes de commercialisation des produits agricoles relève sous certains aspects d'un processus d'intégration marchande. L'apprentissage collectif de l'acte marchand qui se noue au sein des groupes de marché laisse sans doute entrevoir l'émergence de formes économiques plus performantes (Tyuiénon, 2004). Le développement de l'horticulture ornementale, filière dans laquelle le marchand semble prendre une place déterminante dans les efforts de structuration collective, est un exemple de ces apprentissages à l'œuvre.

Pour autant, ce mouvement d'abstraction marchande reste limité. Le salariat peut en effet s'articuler au système d'obligations sociales; il implique un niveau d'abstraction marchande finalement moindre que celui requis par la création d'une entreprise. La faiblesse du tissu des entreprises localisées en tribu ou dirigées par une personne habitant en tribu en témoigne (Sourisseau, 2008). Les données disponibles sur les aides aux activités économiques montrent les difficultés des opérateurs kanak à répondre aux incitations financières, pourtant nombreuses, qui leur sont faites (ibid.). C'est là un signe de la résistance à l'expansion des logiques strictement marchandes, que confirme la très rare spécialisation des ménages dans une activité donnée. Cependant, une partie de ces freins fait écho à des contraintes spécifiques, souvent dissociées du fonctionnement social des tribus : ils renvoient à la longue exclusion des Kanak de secteurs entiers de l'économie.

\section{Quelques traits marquants des processus d'identification sociale}

L'identification sociale permet de maîtriser le développement de l'échange marchand au sein de la société kanak, où la priorité reste donnée à l'autoconsommation et au partage, parfois au détriment de l'accumulation. Elle vise ainsi à contrecarrer les effets jugés les plus déstructurants du marché, à travers des mécanismes spécifiques comme le contrôle collectif des initiatives individuelles (la gestion coutumière du foncier) ou les sollicitations pour utiliser, à des fins communautaires, des outils de production a priori destinés à une utilisation marchande (véhicules, engins, etc.). Le choix de vivre en tribu, qui renvoie à des préférences individuelles mais aussi à des structures collectives et symboliques - lien à la terre et à l'histoire -, limite de fait l'intégration économique. L'individu s'éloigne ainsi des circuits commerciaux et des centres de décisions, ce qui diminue l'inscription des rapports sociaux dans une logique concurrentielle et contribue à en préserver la force.

Plus largement, la justification sociale des contrôles collectifs de l'échange marchand renvoie à « la coutume» en tant que corps de règles définissant les droits et obligations des individus dans l'organisation communautaire. Celle-ci ne saurait être restreinte aux seules interactions avec le marché. Elle apparaît plus globalement comme l'instrument central de régulation des échanges fondés sur la redistribution et la réciprocité définis par Polanyi (1957). Les enquêtes menées insistent sur les fortes hiérarchies internes aux tribus induites par ce corps de règles, mais aussi sur les solidarités qu'il crée et entretient. Le processus d'identification sociale s'exprime par l'ensemble des recompositions sociales à l'œuvre, qui contribuent à faire perdurer, en les adaptant, ces hiérarchies et ces solidarités. La réorganisation des conseils des anciens, la gestion des terres coutumières autorisant de nouvelles formes de cooptation, avec notamment une plus grande implication des clans, la monétarisation d'une partie des échanges coutumiers pour ne pas en exclure les salariés ou encore les dynamiques récentes dans le domaine de la culture sont autant d'illustrations de ces recompositions.

\section{Quels instruments pour accompagner ces processus?}

Les politiques de la province Nord, visant explicitement à laisser aux individus et aux communautés le choix de leur développement et à leur donner les moyens de faire ce choix (Présidence PN, 1999), se heurtent à la complexité des liens entre marchand et non-marchand. En effet, les formes d'hybridation identifiées ici induisent diversité et variabilité dans les pratiques et les dynamiques économiques et sociales. Elles induisent notamment des formes d'organisation inédites que les politiques publiques peinent à accompagner. Le bilan mitigé des programmes d'aides mis en place (Sourisseau, 2008), surtout du point de vue de la durabilité de l'activité financée et des performances économiques, en témoigne.

Il n'y a pas de recette face à une telle situation. Il conviendrait peut-être d'élargir l'éventail des aides en distinguant plus clairement celles visant des activités à vocation exclusivement marchande de celles visant à 
améliorer des activités liées au processus d'identification, tout en améliorant les passerelles entre elles. Il semble aussi opportun de continuer à renforcer les approches de développement territorial, susceptibles de dépasser les logiques des approches filières. Cela suppose de positionner la réflexion stratégique sur les interactions entre les dynamiques économique et sociale à l'œuvre sur un territoire donné, et d'y articuler une participation plus affirmée des acteurs locaux aux processus de décision.

La traduction de ces propositions en politiques publiques est ardue. Pour faciliter la prise en compte par les politiques publiques de ces combinaisons originales, entre marchand et non-marchand, il convient peut-être d'admettre qu'une part d'apprentissage et de tâtonnement est nécessaire. Malgré les exigences de résultats rapides et visibles auxquels les services de développement sont soumis, le développement local commence probablement par la reconnaissance de cette part d'inconnu.

\section{Références}

Aquino, P. d', 2009. La participation comme élément d'une stratégie globale d'intervention : l'approche «gestion autonome progressive», Cahiers Agricultures, 18, 5, 433-440.

Barrau, J., 1956. L'Agriculture vivrière autochtone de la NouvelleCalédonie, Nouméa, Commission du Pacifique Sud.

Barrau, J., 1983. Les Hommes et leurs aliments : esquisse d'une histoire écologique et ethnologique de l'alimentation humaine, Paris, Messidor/Temps actuels.

Bouard, S., d'Aquino, P., Sourisseau, J.-M., Pascutto, T., 2006. Enjeux sociaux, enjeux de développement : expérimentations de méthodes participatives spécifiques pour favoriser les transformations sociales et l'adaptation des modèles de développement à la Nouvelle-Calédonie. Rapport IAC/SRDL, Koné.

Bouard, S., d'Aquino, P., 2007. Une méthode participative pour discuter l'évolution des pratiques de commercialisation des Mélanésiens, Cahiers Agricultures, 16, 6, 491-495.

Castel, O., 2003. La Dynamique institutionnelle de l'économie populaire solidaire dans les pays du Sud, Cahier de la Chaire de recherche en développement des collectivités, série Comparaisons internationales, 4.

Djama, M., 1999. Transformations agraires et systèmes ruraux mélanésiens en Grande Terre de Nouvelle-Calédonie, Journal d'agriculture traditionnelle et de botanique appliquée, 41, $1,201-224$.

Reçu le 10 décembre 2008. Accepté le 2 mars 2010.
Freyss, J., 1995. Économie assistée et changement social en NouvelleCalédonie, Paris, Presses universitaires de France.

Gaillard, C., Sourisseau, J.-M., 2009. Système de culture, système d'activité(s) et rural livelihood : enseignements issus d'une étude sur l'agriculture kanak (Nouvelle-Calédonie), Journal de la Société des océanistes, 129, 279-294.

Godin, P., 2000. Organisations sociales, in Orso, F. (Ed.), Chroniques du pays kanak, Nouméa, Planet Mémo, t. 1, 168-188.

Granovetter, M., 1985. Economic action and social structure : The problem of embeddedness, American Journal of Sociology, 91, 3, 481-510.

Groupe Polanyi, 2008. La Multifonctionnalité de l'agriculture : une dialectique entre marché et identé, Versailles, Quae.

Guiart, J., 1999. Les Mélanésiens devant l'économie de marché : du milieu du XIX ${ }^{\mathrm{e}}$ siècle à la fin du millénaire, Nouméa, Le Rocherà-la-Voile.

Haudricourt, A.-G., 1964. Nature et culture dans la civilisation de l'igname : l'origine des clones et des clans, L'Homme, 4, 1, 93-104.

ISEE, 2005. Tableaux de l'économie calédonienne 2004, Nouméa, TEC.

Le Velly, R., 2002. La notion d'encastrement : une sociologie des échanges marchands, Sociologie du travail, 44,1, 37-53.

Leblic, I., 1993. Les Kanak face au développement : la voie étroite, Grenoble, Presses universitaires de Grenoble / Nouméa, ADCK.

Lelbic, I., 2002. Ignames, interdits et ancêtres en NouvelleCalédonie, Journal de la Société des océanistes, 114-115, 115-127.

Mercoiret, M.-R., Djama, M., et al., 1999. Dynamiques rurales et développement régional dans la province nord de NouvelleCalédonie. Rapport CIRAD TERA, Montpellier.

Polanyi, K., 1957. The economy as instituted process, in Polanyi, K., Arensberg, C., Pearson, H.W. (Eds), Trade and Markets in the Early Empires : Economies in History and Theory, Clencoe, The Free Press, 243-270.

Polanyi, K., Arensberg, C., Pearson, H.W. (Eds), 1957. Trade and Markets in the Early Empires : Economies in History and Theory, Clencoe, The Free Press.

Présidence PN, 1999. Les Politiques publiques de la province Nord, Koné, Présidence de la province Nord.

Sabourin, É., Tyuienon, R., 2007. Produits, monnaie et bingo : les marchés ruraux en Nouvelle-Calédonie entre échange et réciprocité, Revue du Mauss, 29, 301-327.

Sourisseau, J.-M. (Ed.), 2008. Les Sociétés locales face aux défis du développement économique, Nouméa, Grain de sable.

Tyuiénon, R., 2004. L'Igname et l'argent. Mémoire de DHEPS, Université Paris III, Paris. 